\title{
Changes in ACPA Fc glycosylation patterns prior to RA onset
}

Researchers in the Netherlands have detected distinct Fc glycosylation patterns on anti-citrullinated protein antibodies (ACPA) prior to the onset of rheumatoid arthritis (RA). "Galactose and sialic acid residues on ACPA are very similar to those on total IgG up until around 3 months prior to disease onset," explains the lead author of the paper Hans U. Scherer, "at which time ACPA start to lose these residues." Importantly, the investigators found that the reduction in galactose and sialic acid-bearing ACPA was only evident in those patients who went on to develop RA, not those who developed undifferentiated arthritis (UA). Scherer says "the absence of sialic acid and galactose residues is generally considered to render IgG antibodies proinflammatory."

Inflammation is central to the pathogenesis of RA, and ACPA can be used as biomarkers for early diagnosis. Despite this association there is no clear data that ACPA directly contribute to the progression of disease, and most $\mathbf{4}$...the researchers compared Fc glycosylation patterns of total serum IgG1 and ACPA-lgG1... 77

knowledge of the effect of antibody Fc glycosylation comes from monoclonal antibody or mouse studies. For this reason, the researchers compared Fc glycosylation patterns of total serum IgG1 and ACPAIgG1 in order to determine the time frame of Fc glycosylation relative to the onset of RA. They took serum samples from patients with arthralgia $(n=183)$ at various intervals following initial medical consultation with a rheumatologist (baseline). After a median time of 12 months (IQR 6-24), 105 patients went on to develop arthritis, (RA =48, UA =57). Total serum IgG and ACPA were isolated by antigen affinity chromatography and trypsin digests were analysed by mass spectrometry for Fc glycosylation, including galactosylation, sialylation and fucosylation.
The reduction in galactose and sialic acid residues 3 months prior to RA onset coincided with an increase in the erythrocyte sedimentation rate, an early sign of increased systemic inflammation. The researchers also found that, unlike the late change in galactose and sialic acid patterns, "ACPA already carry more fucose on the Fc tail in the years preceding disease development." Scherer hypothesises that "it could be possible to render ACPA immunosuppressive rather than pathogenic." If alteration of Fc glycosylation patterns can be implicated in the pathogenesis of RA "it might become possible to develop very early interventions," he concludes.

Nicholas J. Bernard

Original article Rombouts, Y. et al. Anti-citrullinated protein antibodies acquire a pro-inflammatory Fc glycosylation phenotype prior to the onset of rheumatoid arthritis. Ann. Rheum. Dis. doi:10.1136/ annrheumdis-2013-203565 


\section{CORRECTION}

Rheumatoid arthritis: Changes in ACPA Fc glycosylation patterns prior to RA onset

Nicholas J. Bernard

Nat. Rev. Rheumatol. advance online publication 29 October 2013; doi:10.1038/nrrheum.2013.162

In the original version of this article, the first paragraph incorrectly referred to

'galactose-acid' instead of 'galactose'. This error has now been corrected in the HTML and PDF versions of the article. 